LEWIN, R. A. (1951). J. gen. Microbiol. 5, 926-929.

\title{
Isolation of Sexual Strains of Chlamydomonas
}

\author{
By R. A. LEWIN \\ Osborn Botanical Laboratory, Yale University, Nerw Haven, Connecticut, U.S.A.
}

SUMMARY: The zygospores of several species of Chlamydomonas present in dry soil are very resistant to dehydrating agents. Based on this resistance a method for the isolation of sexual strains has been developed, and two homothallic and three heterothallic species of Chlamydomonas have been obtained in pure culture.

A unicellular green alga which can grow without difficulty in pure culture, and which can be induced to undergo a complete sexual life cycle under experimental conditions, would offer possibilities as a suitable organism for an attack on problems in biochemical genetics for which Neurospora and similar heterotrophs are unsuited. Mating strains of a few species of Chlamydomonas have been isolated (Moewus, 1933; Gerloff, 1940; Smith, 1946; Bold, 1949; Lewin, 1949), but for various reasons none has proved altogether satisfactory. Several workers experienced difficulty in obtaining sexual strains when random isolates of Chlamydomonas from soil were paired and examined for mating reactions. Schreiber (1925) reported a lack of success when as many as 70 clones were tested in this way. An easier method was therefore sought and the following procedure, based chiefly on the differential sensitivity of spores and vegetative cells to desiccation, was devised.

Zygospores of $C$. moewusii Gerloff are only produced as a result of sexual fusion of gametes; this is probably true also for most species in this genus. Such spores, which are only slightly more resistant to heat than are vegetative cells, are nevertheless remarkably resistant to desiccation. The walls are so impermeable that spores may remain viable after immersion for a week in acetone, whereas this treatment kills vegetative cells within a few seconds. Bold (1949) isolated mating types of C. chlamydogama Bold from air-dry soil; Smith (1948) stated that from his experience 'dry soil is a better source of sexual material than is damp soil'. It seems probable that the mating strains obtained by these authors arose from such drought-resistant zygospores.

\section{METHOD}

An enriched soil culture containing vegetative cells and zygospores is treated with acetone. From surviving spores a crude culture is grown. This consists of algal strains presumably capable of again producing zygospores. Pure cultures of these can be obtained by the usual method of dilution streaking on agar. When tested, they may prove either to be homothallic, producing zygospores within the clone, or they may exhibit heterothallism, and only form zygospores when mixed with cells from a culture of opposite mating type. Finally, bacteria-free cultures can be obtained. Details of a preliminary experiment using this method are given below. 


\section{Experimental procedure}

Special difficulties in temperature control arise when attempting laboratory culture under artificial illumination of algae sensitive to heat. As sources of inocula, therefore, soils were chosen from comparatively warm environments, ten from Arizona (from a collection made in 1950 by Dr John Reeder, Osborn Botanical Laboratory) and one from a heated greenhouse in New Haven, Connecticut. These samples were treated in the following manner.

Preparation of enrichment cultures. About $2 \mathrm{~g}$. soil were put into $30 \mathrm{ml}$. of a mineral medium satisfactory for the rapid growth of Chlamydomonas and other algae. This medium had the following composition: $\mathrm{Ca}\left(\mathrm{NO}_{3}\right)_{2} \cdot 4 \mathrm{H}_{2} \mathrm{O}$, $1.0 \mathrm{~g} . ; \mathrm{MgSO}_{4} .7 \mathrm{H}_{2} \mathrm{O}, 0.2 \mathrm{~g}$.; $\mathrm{K}_{2} \mathrm{HPO}_{4}, 0.2 \mathrm{~g}$.; trace elements (see Burkholder $\&$ Nickell, 1949). The use of any organic supplement such as soil extract was avoided, in order to discourage the growth of micro-heterotrophic organisms, such as $C$. chlamydogama (Hutner \& Provasoli (in press)), the organic growthsubstance requirements of which would have to be determined before critical physiological studies could be undertaken.

The flasks so prepared were illuminated under batteries of 'daylight' fluorescent lamps at $c .500$ f.c. intensity, at $23^{\circ}$, for 2 weeks. During this period a dense growth of mixed algae developed, and zygotes were formed by such species as were capable of growth and of the production and fusion of gametes under these conditions.

Acetone treatment. At the end of a fortnight, the contents of each flask were mixed by shaking. Five ml. of the algal suspension was mixed with an equal volume of acetone, allowed to stand for $10 \mathrm{~min}$., and then filtered. The residue was drained and dried in air for $\mathbf{2 0}$ min., to permit evaporation of the acetone, and then rinsed into flasks containing $30 \mathrm{ml}$. of sterile mineral medium, which were then incubated and illuminated as before.

Isolation of strains derived from acetone-resistant zygospores. After illumination for 11 days, seven of these flasks showed abundant growth of unicellular green algae, presumably derived from acetone-resistant zygospores. This period of growth was not extended, since it is preferable for most experimental purposes to work with species in which there is not a prolonged period of zygospore dormancy, as there is normally in C. moerousii (Lewin, 1949). With a flamed bacteriological loop, streaks were made from each of the seven flasks on Petri plates containing mineral medium solidified with $1 \%$ agar. These plates, kept inverted to eliminate condensation of water on the lid, were incubated under continuous illumination.

Within a week, colonies were visible to the naked eye. Five well-isolated colonies were selected on each plate, and transferred to tubes containing c. $5 \mathrm{ml}$. of sterile mineral medium. (One of the plates had two types of colonies: the smaller proved to consist of Chlorococcum sp., and will not be considered further here.) After 5 days of illumination, each tube contained a fairly heavy suspension of algal cells of the Chlamydomonas type. The period of growth was kept short and the medium used simple, since species which manifest a sexual response only after a long period of vegetative 
development, or under specifically altered nutrient conditions, were not desired.

Mating tests. Within each set from the seven flasks, the five supposedly clonal cultures from the selected colonies were then tested for mating in all combinations in checker-board fashion, $5 \times 5$, by mixing drops directly on the surface of a mineral agar plate. The drops were allowed to fall from pipettes. While there was no attempt at aseptic technique at this stage, the pipettes stood in boiling water between transfers, and care was taken to eliminate involuntary cross-contamination of algae. The use of plates prepared 2 days previously, in which the agar surface had dried somewhat, decreased the danger of fusion between neighbouring droplets. The plates were illuminated for 5 days.

In the author's experience, immediate microscopic examination of such mixtures for the presence of clumping or pairing cells was very time-consuming. As mating had in all cases to be confirmed by the formation of zygospores, no preliminary microscopic examination was made, and ultimate spore-formation was used as the criterion for sexual activity. One Petri dish served for each set, the positions of the various combinations being oriented with the help of a guide mark on the base of the plate.

After 5 days on each plate twenty-five green patches were then apparent, comprising colonies of the original five clones and, in duplicate series, the ten possible paired combinations. These colonies were scanned under a magnification of $\times 100$, and scored for the presence or absence of zygospores. Samples were also mounted on slides and checked under a higher magnification.

Preparation of bacteria-free cultures. The cultures which were considered of interest, after testing for sexual reactions as above, were obtained in bacteriafree condition by dilution streaking on agar plates. (One culture, which by itself produced zygospores when first isolated, proved on subsequent examination to have been a mixture of complementary mating types.) Cultures were checked for bacterial contamination in a liquid medium containing glucose (1\%) and Bacto-tryptone (Difco) $(0.5 \%)$, and have since been maintained on agar slants containing minerals, sodium acetate $(0.1 \%)$ and Bacto-tryptone $(0.5 \%)$. From such stocks, liquid cultures in mineral medium were prepared, and rechecked for mating behaviour. In addition, cultures from different soil samples were tested for mating with one another, in order to establish possible specific identities.

\section{RESULTS}

The alga obtained from New Haven greenhouse soil proved to be homothallic, as did one from Arizona. Of the five others which survived the acetone treatment, one yielded no zygospores in any mating combination (even if both mating types had been present, mating conditions may have been otherwise unfavourable). The other four showed typical heterothallic responses. Two isolates from neighbouring sites proved cross-fertile when suitably combined.

Thus mating types of three heterothallic species of Chlamydomonas, and cultures of two homothallic species, were obtained from eleven soil samples, demonstrating the practicability of the method described. 
I am indebted to Prof. G. M. Smith of Stanford University for much valuable assistance by correspondence. This investigation was carried out during tenure of a Theresa Seessel Research Fellowship.

\section{REFERENCES}

BoLd, H. C. (1949). The morphology of Chlamydomonas chlamydogama sp.nov. Bull. Torrey bot. $\mathrm{Cl}$. 76, 101.

Burkholder, P. R. \& Nickeld, L. G. (1949). Atypical growth of plants. I. Culture of virus tumors of Rumex on nutrient agar. Bot. Gaz. 110, 426.

GerlofF, J. (1940). Beiträge zur Kenntnis der Variabilität und Systematik der Gattung Chlamydomonas. Arch. Protistenk. 94, 311.

Hutner, S. H. \& Provasoli, L. (in press). The Phytoflagellates. In Biochemistry and Physiology of the Protozoa. Ed. A. Lwoff. New York: Academic Press Incor.

Lewre, R. A. (1949). Germination of zygospores in Chlamydomonas. Nature, Lond., 164, 543.

Moewus, F. (1933). Untersuchungen über die Sexualität und Entwicklung von Chlorophyceen. Arch. Protistenk. 80, 469.

Schreiber, E. (1925). Zur Kenntnis der Physiologie und Sexualität höherer Volvocales. Z. Bot. 17, 337.

Sмiтн, G. M. (1946). The nature of sexuality in Chlamydomonas. Amer. J. Bot. 33, 625.

Smith, G. M. (c. 1948). Methods for obtaining and culturing sexual strains of Chlamydomonas. Unpublished MS.

(Received 26 April 1951) 